\title{
Recognising, supporting and understanding Autistic adults in general practice settings
}

CPD

Pia Bradshaw, Claire Pickett, Mieke L van Driel, Katie Brooker, Anna Urbanowicz

Pia Bradshaw is Autistic and attention deficit hyperactivity disorder (ADHD) and an autism health researcher and PhD student in Medical Sciences, and Claire Pickett is an Autistic general practitioner.

\section{Background}

The health and wellbeing of Autistic people are well below those of their non-Autistic counterparts. With studies showing a reduced life expectancy and poor physical and mental health, it is important that healthcare professionals strive towards improving the lives of Autistic people by recognising those who are undiagnosed and/or misdiagnosed and providing meaningful support.

\section{Objective}

The aim of this article is to empower general practitioners (GPs) to feel comfortable in recognising potentially undiagnosed Autistic adults. The authors aim to further the understanding of the Autistic experience, including frequently co-occurring physical and mental health conditions, and to give GPs some tools to make the experience of accessing healthcare less problematic for Autistic people.

\section{Discussion}

The healthcare experiences of Autistic adults will be greatly improved with a knowledgeable, understanding and accepting GP as the cornerstone of their care.
MOST GENERAL PRACTITIONERS (GPS) will have several known Autistic patients attending their practice. This includes patients who may have previously been diagnosed with Asperger's syndrome, a diagnostic term that no longer appears in the latest version of the Diagnostic and statistical manual of mental disorders, fifth edition. ${ }^{1}$ These patients may have very outwardly obvious Autistic traits and may fit the practitioner's understanding of the characteristics of a stereotypical Autistic person. Media portrayals of autism often will reinforce a stereotypical understanding of autism, such as that Autistic adults are incapable of holding conversations and making eye contact, having a full-time a job or having a family. These stereotypes about autism and how people who are Autistic look and behave have caused many people to remain undiagnosed, misdiagnosed and misunderstood. ${ }^{2}$

With current data indicating that the prevalence of autism is one in 59 people, ${ }^{3}$ it is likely many GPs have undiagnosed Autistic people among their patient cohort. Indeed, some GPs are Autistic themselves. ${ }^{4}$ Having lived their childhood in an era before the current understanding of autism, the fact that they are Autistic may not have been recognised. Autistic people, whether diagnosed or not, are often struggling with employment, relationships, finances and poor physical and mental health. ${ }^{5,6}$ They have not had the benefit of the understanding

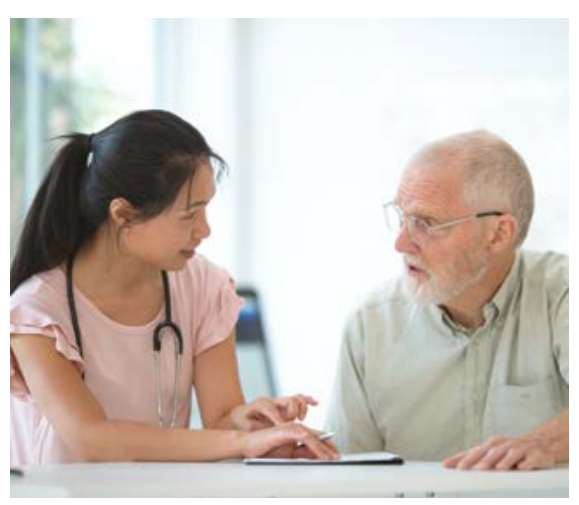

of educators, employers, doctors and society because their difficulties were not recognised as autism. The result is a group of people who are attempting unaided to succeed at the duties and responsibilities of a non-Autistic person, unaware of why they are finding these things difficult. ${ }^{7}$

Autistic people have a nine-fold increased risk of suicidality. ${ }^{8}$ The average life expectancy of an Autistic person is 54 years, well below that of the general population. ${ }^{8}$ Unfortunately, the limited ability of healthcare professionals to identify Autistic people, particularly Autistic women, can result in the accumulation of multiple incorrect diagnoses - such as personality disorder, depression and intellectual disability prior to receiving an autism diagnosis. ${ }^{2}$ As a result, appropriate support is not being offered, and sometimes incorrect treatment is being implemented. ${ }^{2,7}$

There is an opportunity to change these health outcomes by understanding life from the perspective of Autistic people and addressing the specific challenges and barriers that are faced by patients in daily life and in medical consultations. This improves GPs' ability to implement preventive medical strategies that may otherwise be overlooked. In addition, knowing how Autistic people experience the world will make it easier for GPs to build a better relationship with their patients and be also able to recognise those who are unknowingly Autistic. 


\section{How to know if an adult patient is an undiagnosed Autistic person}

Many Autistic people will do their best to fit the expectations of how a patient should behave (eg smiling, making eye contact - sometimes intently - and being overly cautious, polite or apologetic). This effort is called camouflaging or masking. ${ }^{9}$ This makes the person appear as though they are the same as a non-Autistic person. Autistic people subconsciously do this because being their natural selves has often resulted in disapproval or rejection from others. Most people know what is socially expected of them and, with a great deal of effort, Autistic people will conform to those expectations. ${ }^{9}$ Those who were raised as girls can be especially adept at camouflaging as they have had to survive the increased social demands expected of girls. ${ }^{10}$ Camouflaging is something most Autistic people unconsciously do to a lesser or larger degree depending on the person. ${ }^{9}$ This makes it very difficult to tell if someone is Autistic by spending short periods of time with them, such as in a consultation.

The recognition of the following experiences could be helpful in identifying undiagnosed Autistic adults.

\section{Co-occurring medical conditions}

There are several medical conditions that commonly co-occur with autism (Box 1). ${ }^{9,11,12}$ The presence of some of these co-occurring conditions can lead to a consideration of autism. ${ }^{7}$ In addition, these are important to be aware of with known Autistic patients.

\section{Anxiety and depression In particular, it is important to consider autism in patients with anxiety and depression. ${ }^{9,11}$ These are very commonly experienced and are often a secondary reaction to living life unknowingly as an Autistic person. ${ }^{7}$}

\section{Attention deficit hyperactivity disorder Autism and attention deficit hyperactivity disorder (ADHD) coincide. ${ }^{13}$ Autistic adults are over five times more likely to have an ADHD diagnosis than non-Autistic adults. ${ }^{11}$}

Autistic people have heightened senses A patient, when prompted, may disclose that they find lights too bright, sounds too loud, clothes too scratchy or tight, perfumes overwhelming or foods too strong (or sometimes too weak) in flavour. Negotiating the world with heightened senses, known as sensory sensitivity, is challenging and tiring. Once a person knows they are Autistic, an occupational therapist may be helpful in supporting sensory sensitivities. ${ }^{14,15}$

Heightened senses reflect the neurobiological response to environmental stimuli. These neurological responses can cause some Autistic people to experience difficulty interpreting signals from or changes in their body and expressing this to others. ${ }^{16}$ A patient who presents with pain or symptoms that seem incongruous may be an undiagnosed Autistic person. When exploring symptoms further, it is important to give the patient time to think through their answers and take the person at their word even if the symptoms seem unusual.

\section{Family members}

People will often have a family member, including children, with an autism spectrum condition or ADHD. ${ }^{13}$

\section{LGBTIQA+}

Autistic people more commonly identify as part of the LGBTIQA+ (lesbian, gay, bisexual, transgender, intersex, queer/ questioning, asexual and many other terms [such as non-binary and pansexual]) community than their non-Autistic counterparts. ${ }^{17}$

\section{Social and community-based settings} Interestingly, studies show that communication and understanding between Autistic people is similar, if not better, than between people with a non-Autistic neurology. ${ }^{18}$ The communication difficulties of autism occur when people from differing neurotypes are communicating with each other, such as when an Autistic person and a non-Autistic person are communicating together. ${ }^{18}$

As Autistic people are in the minority at most social gatherings, the onus tends to be placed on the Autistic person to change their body language and manner to suit the non-Autistic people in the room. For this reason, socialising and being out in the community becomes quite tiring. A patient may describe feeling tired after long social gatherings and needing time to recover from them. Conversely, they may have some Autistic friends and family members whose company they love and with whom conversation is easy and intuitive.

If the social gathering is in a bright or noisy environment, the Autistic person will often be overloaded with the effort required to be in a space that is environmentally overstimulating. Socialising under these circumstances may be manageable but becomes overwhelming after a while. It is helpful to ask a patient how they feel at social gatherings and other busy settings such as shopping centres and grocery stores. Do they sometimes feel misunderstood or struggle to connect with others? Do they enjoy large or busy social settings? Do they need some time alone to recover afterwards? Do they use drugs or alcohol to cope during such events or after such interactions to relax and regulate?

\section{Fatigue}

Tiredness is a very non-specific but common presenting symptom in general practice. Being Autistic is exhausting. ${ }^{14,19}$ The brain is working overtime to interpret others and block out unwanted sensory input. ${ }^{14}$ It can be helpful to ask

\section{Box 1. A non-exhaustive list of medical conditions that commonly co-occur with autism}

\footnotetext{
- Anxiety ${ }^{10,11}$

- Depression ${ }^{10,11}$

- Attention deficit hyperactivity disorder $r^{10,11,13}$

- Migraine with aura ${ }^{26}$

- Eating disorder or food sensitivities ${ }^{10}$

- Post-traumatic stress disorder ${ }^{27}$

- Sleep apnoea ${ }^{10,12}$

- Gastrointestinal disorders $s^{10-12}$

- Epilepsy ${ }^{11,26}$

- Hypermobility spectrum disorders/ Ehlers-Danlos syndromes ${ }^{10}$

- Fibromyalgia ${ }^{10}$

- Obsessive-compulsive disorder ${ }^{11}$

- Autonomic dysfunction ${ }^{28}$
} 
patients about what makes them feel tired. Together, it may be possible to discern whether the ordinary demands of day-to-day life indicate problems with executive dysfunction, working memory or sensory sensitivities. For example, does tiredness occur after a prolonged period of interacting with others or when exposed to excessive noise, movement or light?

\section{Childhood and teenage years}

Early experiences will often suggest that a patient is potentially Autistic. A patient may be able to recount if they were a child or teenager who fitted in well with their same-aged peers or not. There can be a history of bullying for their differences. Teenage experiences may include depression, anxiety, self-harm, substance abuse, eating disorders, inability to keep up with workloads and burning-out or melting down if overwhelmed by the stress of managing daily demands. These experiences may continue into adulthood.

\section{Organisation}

The patient who is always rushing into their appointments in a flurry of apologies or forgetting to have their blood tests may just be a busy person. However, if this is happening in conjunction with other potential indicators of autism, it may be significant. Executive function is the term used for the brain's ability to manage life. Modern living demands timely replies to emails, the keeping of appointments, adhering to schedules and commitments. Autistic people have challenges with executive function, which understandably makes dealing with these necessities extremely difficult and demanding. ${ }^{10}$

On the other hand, some Autistic people may be very organised and use this as a coping strategy to manage unforeseen circumstances or anxiety regarding healthcare visits. For example, an Autistic patient may arrive early for an appointment.

\section{How to improve general practice to facilitate access to healthcare for Autistic patients \\ When caring for an Autistic patient, it is important to bear in mind that waiting}

rooms are very bright and busy places. This may cause patients to be overloaded by the time they come into the consulting room, which can affect their ability to communicate. It is reasonable to offer Autistic patients the option to wait in their car, outside or in a quiet room within the practice, and have reception staff text them when the GP is ready to see them (Box 2).

It is imperative to build trust with Autistic patients through greater autism awareness and demonstrating autism acceptance. After getting to know and trust their GP, a patient may feel comfortable to camouflage less. When caring for an Autistic person, it is important to avoid showing disapproval of the way they express themselves and be mindful of dismissing their concerns, no matter how minor or insignificant they may appear. For example, some Autistic people may ask more detailed questions of the GP than the GP might expect, or they may be quite direct. It is important to allow the patient the time to talk through their concerns and patiently work through any questions one at a time. Occasionally a long consultation may be needed to avoid running overtime. Reading between the lines should be avoided. Some Autistic people will struggle if GPs use analogies, irony or sarcasm, so it is best to use a straightforward communication style.

The healthcare system is particularly dependant on a patient's ability to manage their appointments, collect scripts, take daily medications, etc. A non-judgemental attitude towards executive function slip-ups will help break down healthcare barriers. A GP could offer to make phone calls on behalf of the patient to facilitate radiology or specialist appointments, or ask reception staff to do so and write down any further instructions for them.

It is important to ensure non-medical staff are sensitive to Autistic patients. These staff members can often inadvertently be significant barriers to an Autistic person navigating access to care.

Having a review with Autistic patients on a regular basis is a good way to keep an eye on their healthcare. If a patient has not presented in a while, it does not mean that all is well.

\section{Box 2. Practice tips for caring for Autistic adults}

- When discussing autism, use strengths-based language that promotes acceptance and avoid deficits-based descriptions.

- Avoid dismissing or assuming that genuine health concerns are part of being inherently Autistic.

- Invite Autistic patients to share their experience of autism and avoid making assumptions about their Autistic experience.

- Allow time for the patient to answer questions and do not read between the lines/make assumptions about their capacity or challenges on the basis of outward appearances.

- Use a straightforward communication style, avoid analogies/irony/sarcasm and use short sentences.

- Be open to different modes of communication.

- Support non-medical staff to be sensitive to Autistic needs and ensure patients feel welcome and at ease. This is particularly relevant for reception staff, as they are the first point of contact.

- Create an action plan for next steps with the patient to support working memory challenges.

- Offer quiet places for the patient to wait (eg separate room, outside or in their car).

- Allow patients the means to book appointments, including longer appointments, beyond the phone or in person, such as using booking apps or websites without needing to call.

- Be mindful of running late and, where possible, indicate wait time or position in queue.

- Be understanding of late or missed appointments or other executive function slip-ups.

- Send reminders about appointments.

- Offer support to facilitate making appointments for referrals either at the time of the appointment or by following this up later.

- Schedule regular/standing appointments to ensure patients are receiving preventive care. 
Useful and readily available local and international resources for providing care to Autistic adults are listed in Table 1.

\section{What to do next}

If a GP suspects their patient may be Autistic, it is best to sensitively ask the patient their thoughts about the subject. It is important that the language used to talk about autism is strengths based and promotes autism acceptance..$^{20}$ Not everyone will want to pursue the possibility of autism further, but many will.

The patient can complete the Autism Spectrum Quotient. ${ }^{21}$ This is a 50-item questionnaire, accessible online at home. This is a screening test, not a diagnostic tool, that measures the presence of Autistic traits. The results of the test will indicate the probability of autism and can help the patient decide if formal testing is something they would like to pursue.

Formal testing in adulthood is usually performed by a psychologist or psychiatrist who is experienced working with Autistic adult. For adults, particularly those who identify as female, it is necessary to see a psychologist or psychiatrist who has significant experience working with Autistic adults and with the female presentation of autism. Cost, relevant expertise, location and wait time of a formal assessment can be a barrier as there are few funded adult assessment clinics in Australia and only a handful of psychiatrists or psychologists who are actually Autistic themselves. ${ }^{22}$ Post-diagnostic support in the form of autism-based counselling sessions is an important follow-up for the patient, as is connecting with other Autistic people. ${ }^{23-25}$

\section{Conclusion}

Autistic people contribute to the diversity of humanity and are an important part of society. It is important to remember that GPs view their patients through the lens of their own experience of the world. Non-Autistic GPs may find it hard to relate to the experiences of an Autistic person. The most important tool is listening, respecting and learning from the patient's lived experience as an Autistic person.
In particular, GPs should try to listen to the words a person is saying and avoid language, communicative abilities or preference, facial expressions, intonation or eye contact. It is hoped that this article will help GPs feel comfortable in meeting the healthcare needs and communicative differences of their Autistic patients to improve these patients' wellbeing, health outcomes and interactions.

\section{Key points}

- It is likely that many GPs have undiagnosed Autistic adults among their patient cohort.

- There are certain conditions, such as anxiety, depression and ADHD, that commonly co-occur with autism.

- Correctly identifying undiagnosed Autistic adults can facilitate access to appropriate supports.

- If there are suspicions that a patient is Autistic, it may be helpful to sensitively talk to them about it. making assumptions on the basis of body

- It is important that GPs support patients to access a formal autism assessment if this is the patients' wish.

\section{Authors}

Pia Bradshaw BIS (Hons), MA, Queensland Centre for Intellectual and Developmental Disability (QCIDD), Mater Research Institute, The University of Queensland, and The Cooperative Research Centre for Living with Autism (Autism CRC), Qld

Claire Pickett MBBS, DipRACOG, Certificate of Sexual and Reproductive Health, General Practitioner, Ballarat Community Health Lucas Facility, Vic

Mieke L van Driel MD, MSc, PhD, FRACGP, General Practitioner and Emeritus Professor, Mayne Academy of General Practice and Primary Care Clinical Unit, Faculty of Medicine, The University of Queensland, QId

Katie Brooker PhD, BHIthSc (Hons), Postdoctoral Research Fellow, Queensland Centre for Intellectual and Developmental Disability (QCIDD), Mater Research Institute, The University of Queensland, and The Cooperative Research Centre for Living with Autism (Autism CRC), Qld

Anna Urbanowicz PhD, BSc (Hons), Postdoctoral Fellow, Social and Global Studies Centre, School of Global, Urban and Social Studies, RMIT University; Honorary Fellow, Queensland Centre for Intellectual and Developmental Disability (QCIDD), Mater Research Institute, The University of Queensland, QId Competing interests: $\mathrm{PB}$ is Autistic and attention deficit hyperactivity disorder (ADHD) and an autism health researcher and $\mathrm{PhD}$ student in Medical Sciences, and CP is an Autistic GP.

Table 1. Resources for general practitioners to support provision of care to Autistic adults

\begin{tabular}{|c|c|c|}
\hline Resource & Description & URL \\
\hline $\begin{array}{l}\text { Autism } \\
\text { HealthPathways }\end{array}$ & $\begin{array}{l}\text { Online tool used to help make } \\
\text { assessment, management and } \\
\text { referral decisions. Pathways for } \\
\text { autism have been developed by the } \\
\text { HealthPathways Mackay team and } \\
\text { are ready to be localised. }\end{array}$ & $\begin{array}{l}\text { www.healthpathwayscommunity. } \\
\text { org/News/Latest-Community- } \\
\text { News/ArticlelD/3072/ } \\
\text { HealthPathways-Mackay- } \\
\text { puts-the-spotlight-on-Autism- } \\
\text { Spectrum-Disorder }\end{array}$ \\
\hline $\begin{array}{l}\text { AASPIRE } \\
\text { Healthcare } \\
\text { Toolkit }\end{array}$ & $\begin{array}{l}\text { Online toolkit that includes } \\
\text { information, checklists and worksheets } \\
\text { for primary healthcare providers, } \\
\text { Autistic patients and supporters }\end{array}$ & https://autismandhealth.org \\
\hline $\begin{array}{l}\text { My Health Passport } \\
\text { for Autistic people }\end{array}$ & $\begin{array}{l}\text { PDF tool developed by the UK } \\
\text { National Autistic Society to help } \\
\text { Autistic people to communicate their } \\
\text { needs to healthcare professionals }\end{array}$ & $\begin{array}{l}\text { www.autism.org.uk/advice- } \\
\text { and-guidance/topics/physical- } \\
\text { health/my-health-passport }\end{array}$ \\
\hline $\begin{array}{l}\text { NICE clinical } \\
\text { guideline - Autism } \\
\text { spectrum disorder } \\
\text { in adults: Diagnosis } \\
\text { and management }\end{array}$ & $\begin{array}{l}\text { Online guideline providing } \\
\text { information on diagnosis and } \\
\text { managing suspected or confirmed } \\
\text { autism in adults }\end{array}$ & $\begin{array}{l}\text { www.nice.org.uk/guidance/ } \\
\text { cg142 }\end{array}$ \\
\hline $\begin{array}{l}\text { UK Royal College of } \\
\text { General Practitioners } \\
\text { Autistic Spectrum } \\
\text { Disorders Toolkit }\end{array}$ & $\begin{array}{l}\text { Online toolkit that includes } \\
\text { information, checklists and } \\
\text { worksheets for general practitioners }\end{array}$ & $\begin{array}{l}\text { www.rcgp.org.uk/clinical-and- } \\
\text { research/resources/toolkits/ } \\
\text { asd-toolkit.aspx }\end{array}$ \\
\hline
\end{tabular}


Funding: PB and KB are funded by the Cooperative Research Centre for Living with Autism (Autism $\mathrm{CRC}$ ), established and supported under the Australian Government's Cooperative Research Centres Program. AU is funded by RMIT ViceChancellor's Postdoctoral Research Fellowship (Fulbright Scholar).

Provenance and peer review: Commissioned, externally peer reviewed.

\section{Correspondence to:}

pia.bradshaw@uq.edu.au

\section{References}

1. American Psychiatric Association. Diagnostic and statistical manual of mental disorders. 5th edn. Arlington, VA: APA, 2013.

2. Fusar-Poli L, Brondino N, Politi P, Aguglia E. Missed diagnoses and misdiagnoses of adults with autism spectrum disorder. Eur Arch Psychiatry Clin Neurosci 2020. doi: 10.1007/ s00406-020-01189-w.

3. Baio J, Wiggins L, Christensen DL, et al. Prevalence of autism spectrum disorder among children aged 8 years - Autism and developmental disabilities network, 11 sites, United States, 2014. MMWR Surviell Summ 2018;67(6):1-23. doi: 10.15585/mmwr.ss6706a1.

4. Moore S, Kinnear M, Freeman L. Autistic doctors: Overlooked assets to medicine. Lancet Psychiatry 2020;7(4):306-07. doi: 10.1016/S2215 0366(20)30087-0.

5. Wei X, Yu JW, Wagner $M$, et al. Job searching job duration and job loss among young adults with autism spectrum disorder. J Vocat Rehabil 2018;48(1):1-10. doi: 10.3233/JVR-170922.

6. Cooper K, Smith LGE, Russell A. Social identity, self-esteem, and mental health in autism. Eur J Soc Psychol 2017;47(7):844-54. doi: 10.1002/ ejsp.2297.

7. Lai MC, Baron-Cohen S. Identifying the lost generation of adults with autism spectrum conditions. Lancet Psychiatry 2015;2(11):1013-27. doi: 10.1016/S2215-0366(15)00277-1.

8. Hirvikoski T, Mittendorfer-Rutz E, Boman M, Larsson H, Lichtenstein P, Bölte S. Premature mortality in autism spectrum disorder. $\mathrm{Br} J$ Psychiatry 2016;208(3):232-38. doi: 10.1192/bjp. bp.114.160192.

9. Hull L, Petrides KV, Allison $C$, et al. 'Putting on my best normal': Social camouflaging in adults with autism spectrum conditions. J Autism Dev Disord 2017;47(8):2519-34. doi: 10.1007/s10803017-3166-5.

10. Cook B, Garnett M. Spectrum women: Walking to the beat of autism. London, UK: Jessica Kingsley Publishers, 2018.

11. Croen LA, Zerbo O, Qian Y, et al. The health status of adults on the autism spectrum. Autism 2015;19(7):814-23. doi: 10.1177/1362361315577517.

12. Tye C, Runicles AK, Whitehouse AJO, Alvares GA. Characterizing the interplay between autism spectrum disorder and comorbid medical conditions: An integrative review. Front Psychiatry 2019;9:751. doi: 10.3389/fpsyt.2018.00751.

13. Ghirardi L, Brikell I, Kuja-Halkola R, et al. The familial co-aggregation of ASD and ADHD: A register-based cohort study. Mol Psychiatry 2018;23(2):257-62. doi: 10.1038/mp.2017.17.

14. Crane L, Batty R, Adeyinka H, Goddard L, Henry LA, Hill EL. Autism diagnosis in the United Kingdom: Perspectives of autistic adults, parents and professionals. J Autism Dev Disord 2018;48(11):3761-72. doi: 10.1007/ s10803-018-3639-1.
15. Lewis LF. A mixed methods study of barriers to formal diagnosis of autism spectrum disorder in adults. J Autism Dev Disord 2017;47(8):2410-24. doi: 10.1007/s10803-017-3168-3.

16. Jones L, Goddard L, Hill EL, Henry LA, Crane L. Experiences of receiving a diagnosis of autism spectrum disorder: A survey of adults in the United Kingdom. J Autism Dev Disord 2014;44(12):3033-44. doi: 10.1007/s10803-0142161-3.

17. Vetri L. Autism and migraine: An unexplored assocation? Brain Sci 2020;10(9):1-15. doi: 10.3390/brainsci10090615.

18. Haruvi-Lamdan N, Horesh D, Zohar S, Kraus M, Golan O. Autism spectrum disorder and post-traumatic stress disorder: An unexplored co-occurrence of conditions. Autism 2020;24(4):884-98. doi: 10.1177/1362361320912143.

19. Ming X, Patel R, Kang V, Chokroverty S, Julu PO. Respiratory and autonomic dysfunction in children with autism spectrum disorders. Brain Dev 2016;38(2):225-32. doi: 10.1016/j. braindev.2015.07.003.

20. Bradshaw P, Pickett C, van Driel ML, Brooker K, Urbanowicz A. 'Autistic' or 'with autism'? Why the way general practitioners view autism matters. Aust J Gen Pract 2021;50(3):104-09.

21. DuBois D, Ameis SH, Lai MC, Casanova MF, Desarkar P. Interoception in autism spectrum disorder: A review. Int J Dev Neurosci 2016;52:104-11. doi: 10.1016/j. ijdevneu.2016.05.001.

22. Goodman-Scott E, Burgess M, Polychronopoulos G. Counseling adults with sensory processing disorder: An exploratory study. J Ment Health Couns 2020;42(3):234-50. doi: 10.17744/mehc.42.3.04.

23. Crane L, Goddard L, Pring L. Sensory processing in adults with autism spectrum disorders. Autism 2009;13(3):215-28. doi: 10.1177/1362361309103794.

24. Raymaker DM, Teo AR, Steckler NA, et al. 'Having all of your internal resources exhausted beyond measure and being left with no clean-up crew': Defining autistic burnout. Autism Adulthood 2020;2(2):132-43. doi: 10.1089/aut.2019.0079.

25. George R, Stokes MA. Gender identity and sexual orientation in autism spectrum disorder. Autism 2018;22(8):970-82. doi: 10.1177/1362361317714587.

26. Crompton CJ, Ropar D, Evans-Williams CV, Flynn EG, Fletcher-Watson S. Autistic peer-to-peer information transfer is highly effective. Autism 2020;24(7):1704-12. doi: 10.1177/1362361320919286.

27. Baron-Cohen S, Wheelwright S, Skinner R, Martin J, Clubley E. The autism-spectrum quotient (AQ): Evidence from asperger syndrome/high-functioning autism, males and females, scientists and mathematicians. J Autism Dev Disord 2001;31(1):5-17. doi: 10.1023/a:1005653411471.

28. Amaze. Amaze position statement: Assessment and diagnosis. Carlton, Vic: Amaze, 2018. 